\title{
New type of degenerate Daehee polynomials of the second kind
}

\author{
Sunil Kumar Sharma ${ }^{*}$ (D), Waseem A. Khan², Serkan Araci ${ }^{3}$ (D) and Sameh S. Ahmed ${ }^{4}$
}

\section{"Correspondence:}

s.sharma@mu.edu.sa

'Department of Information

System, College of Computer and

Information Sciences, Majmaah

University, Majmaah 11952, Saudi

Arabia

Full list of author information is

available at the end of the article

\begin{abstract}
Recently, Kim and Kim (Russ. J. Math. Phys. 27(2):227-235, 2020) have studied new type degenerate Bernoulli numbers and polynomials by making use of degenerate logarithm. Motivated by (Kim and Kim in Russ. J. Math. Phys. 27(2):227-235, 2020), we consider a special class of polynomials, which we call a new type of degenerate Daehee numbers and polynomials of the second kind. By using their generating function, we derive some new relations including the degenerate Stirling numbers of the first and second kinds. Moreover, we introduce a new type of higher-order degenerate Daehee polynomials of the second kind. We also derive some new identities and properties of this type of polynomials.
\end{abstract}

MSC: 11B83; 11B73; 05A19

Keywords: Daehee polynomials and numbers; Degenerate Daehee numbers and polynomials of the second kind; Generating function; Summation formula

\section{Introduction and preliminaries}

Special polynomials have their origin in the solutions of differential equations under some conditions. Special polynomials can be described in various ways such as by generating functions, by $p$-adic integrals, by recurrence relations, by degenerate versions, and so on.

The degenerate versions of some special numbers and polynomials have been studied by many researchers. The notion of degeneracy provides a powerful tool in defining special numbers and polynomials of their degenerate versions. The most important applications of these polynomials are in the theory of finite differences, analytic number theory, and applications in the classical analysis and statistics. Despite the applicability of special functions in classical analysis and statistics, they also arise in communications systems, quantum mechanics, nonlinear wave propagation, electric circuit theory, electromagnetic theory, and so on.

Recent investigations involving degenerate Daehee polynomials and numbers of the third kind [3], degenerate $\lambda-q$-Daehee polynomials [5], degenerate polyexponential functions and degenerate Bell polynomials [14], degenerate binomial coefficients and degenerate hypergeometric functions [15], new type degenerate Bernoulli numbers [8], degenerate Stirling polynomials of the second kind [19], degenerate poly-Bernoulli numbers and polynomials [18], degenerate Daehee polynomials of the second kind [9], new type de-

(c) The Author(s) 2020. This article is licensed under a Creative Commons Attribution 4.0 International License, which permits use sharing, adaptation, distribution and reproduction in any medium or format, as long as you give appropriate credit to the original author(s) and the source, provide a link to the Creative Commons licence, and indicate if changes were made. The images or other third party material in this article are included in the article's Creative Commons licence, unless indicated otherwise in a credit line to the material. If material is not included in the article's Creative Commons licence and your intended use is not permitted by statutory regulation or exceeds the permitted use, you will need to obtain permission directly from the copyright holder. To view a copy of this licence, visit http://creativecommons.org/licenses/by/4.0/. 
generate Daehee numbers and polynomials [17], some results on degenerate Daehee and Bernoulli numbers and polynomials [20], degenerate Laplace transform and degenerate gamma function [11], some identities on type 2 degenerate Bernoulli polynomials of the second sind [10], some identities for degenerate complete and incomplete $r$-Bell polynomials [21] have been investigated in detail.

We are now in a position to state some special numbers and polynomials, which will be used in the paper.

Let $B_{n}^{(r)}(x)$ be the generalized Bernoulli polynomials of order $r$ given by the following generating function (see $[1,6,12])$ :

$$
\sum_{n=0}^{\infty} B_{n}^{(r)}(x) \frac{t^{n}}{n !}=\left(\frac{t}{e^{t}-1}\right)^{r} e^{x t} \quad\left(r \in \mathbb{C} ; 1^{r}:=1 ;|t|<2 \pi\right) .
$$

In the case where $x=0, B_{n}^{(r)}=: B_{n}^{(r)}(0)$ are called the generalized Bernoulli numbers of order $r$.

The notion of degenerate of the exponential function

$$
e^{z}=\lim _{\lambda \rightarrow 0}(1+\lambda z)^{\frac{1}{\lambda}}
$$

is considered without the limit case. That is, the degenerate of the exponential function $e^{z}$ is equal to $(1+\lambda z)^{\frac{1}{\lambda}}$. It follows that the degenerate of the parameter $z$ is $\frac{\log (1+\lambda z)}{\lambda}$. This idea was first considered for Bernoulli polynomials by Carlitz [1] as follows:

$$
\sum_{n=0}^{\infty} \beta_{n, \lambda}(x) \frac{t^{n}}{n !}=\frac{t}{(1+\lambda t)^{\frac{1}{\lambda}}-1}(1+\lambda t)^{\frac{x}{\lambda}} \quad(\lambda \in \mathbb{R}) .
$$

At the point $x=0$ in (2), $\beta_{n, \lambda}=: \beta_{n, \lambda}(0)$ are called the degenerate Bernoulli numbers.

Let $(x)_{n}$ be the falling factorial sequence given by

$$
(x)_{n}=x(x-1) \cdots(x-n+1) \quad(n \geq 1)
$$

with the assumption $(x)_{0}:=1$.

As is well known, the degenerate Bernoulli polynomials of higher order were considered by Carlitz [1] as follows:

$$
\sum_{n=0}^{\infty} \beta_{n, \lambda}^{(r)}(x) \frac{t^{n}}{n !}=\left(\frac{t}{(1+\lambda t)^{\frac{1}{\lambda}}-1}\right)^{r}(1+\lambda t)^{\frac{x}{\lambda}} .
$$

Obviously,

$$
\lim _{\lambda \rightarrow 0} \beta_{n, \lambda}^{(r)}(x)=B_{n}^{(r)}(x)
$$

which represents the Bernoulli polynomials of higher order.

For $\lambda \in \mathbb{R}$, Kim and Kim [8] defined the degenerate version of the logarithm function, denoted by $\log _{\lambda}(1+t)$, as follows:

$$
\log _{\lambda}(1+t)=\sum_{n=1}^{\infty} \lambda^{n-1}(1)_{n, 1 / \lambda} \frac{t^{n}}{n !}
$$


which is the inverse of the degenerate version of the exponential function $e_{\lambda}(t)$ as

$$
e_{\lambda}\left(\log _{\lambda}(t)\right)=\log _{\lambda}\left(e_{\lambda}(t)\right)=t \text {. }
$$

Note that

$$
\lim _{\lambda \rightarrow 0} \log _{\lambda}(1+t)=\sum_{n=1}^{\infty}(-1)^{n-1} \frac{t^{n}}{n !}=\log (1+t) .
$$

The degenerate polyexponential function (see $[7,14])$ is defined by

$$
e_{\lambda}(x, \delta \mid k)=\sum_{n=0}^{\infty} \frac{(1)_{n, \lambda}}{n !(n+\delta)^{k}} x^{n} \quad\left(k \in \mathbb{N}_{0} \text { and } \delta \in \mathbb{C} \text { with } \Re e(\delta)>0\right) .
$$

The degenerate Stirling numbers of the second kind (see [9]) are defined by

$$
\frac{1}{k !}\left(\log _{\lambda}(1+t)\right)^{k}=\sum_{n=k}^{\infty} S_{1, \lambda}(n, k) \frac{t^{n}}{n !} \quad(k \geq 0) .
$$

Note that

$$
\lim _{\lambda \rightarrow 0} S_{1, \lambda}(n, k)=S_{1}(n, k),
$$

which stands for the Stirling numbers of the first kind given by (see [20])

$$
\frac{1}{k !}(\log (1+t))^{k}=\sum_{n=k}^{\infty} S_{1}(n, k) \frac{t^{n}}{n !}
$$

The degenerate Stirling numbers of the second kind (see $[16,19])$ are given by

$$
\frac{1}{k !}\left(e_{\lambda}(t)-1\right)^{k}=\sum_{n=k}^{\infty} S_{2, \lambda}(n, k) \frac{t^{n}}{n !} \quad(k \geq 0)
$$

It is clear that

$$
\lim _{\lambda \rightarrow 0} S_{2, \lambda}(n, k)=S_{2}(n, k)
$$

which are called the Stirling numbers of the second kind given by means of the following generating function:

$$
\frac{1}{k !}\left(e^{t}-1\right)^{k}=\sum_{n=k}^{\infty} S_{2}(n, k) \frac{t^{n}}{n !} \quad(\text { see }[25])
$$

Roman [24] defined the Bernoulli polynomials of the second kind given by the generating function

$$
\frac{t}{\log (1+t)}(1+t)^{x}=\sum_{n=0}^{\infty} b_{n}(x) \frac{t^{n}}{n !} .
$$


It is worth noting that (cf. [9])

$$
b_{n}(x)=B_{n}^{(n)}(x+1) \quad(n \geq 0) .
$$

The degenerate version of Eq. (9) is given by Kim and Kim [14] as follows:

$$
\sum_{n=0}^{\infty} b_{n, \lambda}(x) \frac{t^{n}}{n !}=\frac{t}{\log _{\lambda}(1+t)}(1+t)^{x}
$$

satisfying

$$
\lim _{\lambda \rightarrow 0} b_{n, \lambda}(x)=b_{n}(x) .
$$

The Daehee polynomials are defined by

$$
\frac{\log (1+t)}{t}(1+t)^{x}=\sum_{n=0}^{\infty} D_{n}(x) \frac{t^{n}}{n !}
$$

In the case where $x=0$ in (11), $D_{n}=: D_{n}(0)$ are called the Daehee numbers (see $[2-4,8,13$, $14,17,18,20,22,23,25])$.

Very recently, Kim et al. [17] introduced a new class of degenerate Daehee polynomials via the following generating function:

$$
\sum_{n=0}^{\infty} d_{n, \lambda}(x) \frac{t^{n}}{n !}=\frac{\log _{\lambda}(1+t)}{t}(1+t)^{x} .
$$

Putting $x=0$ in (12) yields $d_{n}=: d_{n}(0)$, the degenerate Daehee numbers.

By (2) and (12) we get

$$
\begin{aligned}
\sum_{n=0}^{\infty} \beta_{n, \lambda}(x) \frac{t^{n}}{n !} & =\sum_{m=0}^{\infty} d_{m, \lambda}(x) \frac{1}{m !}\left(e_{\lambda}(t)-1\right)^{m} \\
& =\sum_{m=0}^{\infty} d_{m, \lambda}(x) \sum_{n=m}^{\infty} S_{2, \lambda}(n, m) \frac{t^{n}}{n !} \\
& =\sum_{n=0}^{\infty}\left(\sum_{m=0}^{n} S_{2, \lambda}(n, m) d_{m, \lambda}(x)\right) \frac{t^{n}}{n !} .
\end{aligned}
$$

By comparing the coefficients on both sides of (13) we have the following summation formula for the products $S_{2, \lambda}(n, m)$ and $d_{m, \lambda}(x)$ :

$$
\beta_{n, \lambda}(x)=\sum_{m=0}^{n} S_{2, \lambda}(n, m) d_{m, \lambda}(x) \quad(n \geq 0) .
$$

In 2017, Kim and Kim [9] considered the degenerate Daehee polynomials of the second kind defined by

$$
\frac{\log (1+t)}{(1+\lambda \log (1+t))^{\frac{1}{\lambda}}-1}(1+\lambda \log (1+t))^{\frac{x}{\lambda}}=\sum_{n=0}^{\infty} \mathcal{D}_{n, \lambda}(x) \frac{t^{n}}{n !} .
$$


When $x=0$ in (15), $D_{n, \lambda}=D_{n, \lambda}(0)$ stand for the degenerate Daehee numbers of the second kind.

Motivated by the works of Kim and Kim [8, 17], we first define a new type of degenerate Daehee numbers and polynomials of the second kind. We investigate some new properties of these numbers and polynomials and derive some new identities and relations between the new type of degenerate Daehee numbers and polynomials of the second kind and Carlitz's degenerate Bernoulli polynomials.

\section{New type degenerate Daehee polynomials of the second kind}

In this section, we begin with the following definition.

Definition 2.1 Let $\lambda$ be a real number. The new type degenerate Daehee polynomials of the second kind are given by means of the following generating function:

$$
\sum_{n=0}^{\infty} \widetilde{\mathcal{D}}_{n, \lambda}(x) \frac{t^{n}}{n !}=\frac{\log _{\lambda}(1+t)}{\left(1+\lambda \log _{\lambda}(1+t)\right)^{\frac{1}{\lambda}}-1}\left(1+\lambda \log _{\lambda}(1+t)\right)^{\frac{x}{\lambda}} .
$$

In the case where $x=0, \widetilde{\mathcal{D}}_{n, \lambda}=: \widetilde{\mathcal{D}}_{n, \lambda}(0)$ are called the new type degenerate Daehee polynomials of the second kind.

It follows from (16) that

$$
\lim _{\lambda \rightarrow 0} \widetilde{\mathcal{D}}_{n, \lambda}(x)=D_{n}(x), \quad \text { cf. }[2,4,13,22,23] .
$$

Theorem 2.1 Let $n$ be a nonnegative number. Then

$$
\widetilde{\mathcal{D}}_{n, \lambda}(x)=\sum_{m=0}^{n} \beta_{m, \lambda}(x) S_{1, \lambda}(n, m) .
$$

Proof By using (2) and (16) we get

$$
\begin{aligned}
\sum_{n=0}^{\infty} \widetilde{\mathcal{D}}_{n, \lambda}(x) \frac{t^{n}}{n !} & =\frac{\log _{\lambda}(1+t)}{\left(1+\lambda \log _{\lambda}(1+t)\right)^{\frac{1}{\lambda}}-1}\left(1+\lambda \log _{\lambda}(1+t)\right)^{\frac{x}{\lambda}} \\
& =\sum_{m=0}^{\infty} \beta_{m, \lambda}(x) \frac{1}{m !}\left(\log _{\lambda}(1+t)\right)^{m} \\
& =\sum_{m=0}^{\infty} \beta_{m, \lambda}(x) \sum_{n=m}^{\infty} S_{1, \lambda}(n, m) \frac{t^{n}}{n !} \\
& =\sum_{n=0}^{\infty}\left(\sum_{m=0}^{n} \beta_{m, \lambda}(x) S_{1, \lambda}(n, m)\right) \frac{t^{n}}{n !}
\end{aligned}
$$

Therefore by (17) and (18) we complete the proof.

Theorem 2.2 Let $n$ be nonnegative number. Then we have the identity

$$
\widetilde{\mathcal{D}}_{n, \lambda}(x)=\sum_{k=0}^{n} \sum_{l=0}^{k}\left(\begin{array}{l}
n \\
k
\end{array}\right)(x)_{l, \lambda} S_{1, \lambda}(k, l) \widetilde{\mathcal{D}}_{n-k, \lambda}
$$


Proof Recall from (16) that

$$
\sum_{n=0}^{\infty} \widetilde{\mathcal{D}}_{n, \lambda}(x) \frac{t^{n}}{n !}=\frac{\log _{\lambda}(1+t)}{\left(1+\lambda \log _{\lambda}(1+t)\right)^{\frac{1}{\lambda}}-1}\left(1+\lambda \log _{\lambda}(1+t)\right)^{\frac{x}{\lambda}}
$$

By the binomial theorem

$$
\left(1+\lambda \log _{\lambda}(1+t)\right)^{\frac{x}{\lambda}}=\sum_{l=0}^{\infty}\left(\frac{x}{\lambda}\right)_{l} \lambda^{l} \frac{1}{l !}\left(\log _{\lambda}(1+t)\right)^{l}
$$

we have

$$
\begin{aligned}
\sum_{n=0}^{\infty} \widetilde{\mathcal{D}}_{n, \lambda}(x) \frac{t^{n}}{n !} & =\frac{\log _{\lambda}(1+t)}{\left(1+\lambda \log _{\lambda}(1+t)\right)^{\frac{1}{\lambda}}-1} \sum_{l=0}^{\infty}\left(\frac{x}{\lambda}\right)_{l} \lambda^{l} \frac{1}{l !}\left(\log _{\lambda}(1+t)\right)^{l} \\
& =\left(\sum_{m=0}^{\infty} \widetilde{\mathcal{D}}_{m, \lambda} \frac{t^{m}}{m !}\right)\left(\sum_{l=0}^{\infty}(x)_{l, \lambda} \sum_{k=0}^{\infty} S_{1, \lambda}(k, l) \frac{t^{k}}{k !}\right) \\
& =\left(\sum_{m=0}^{\infty} \widetilde{\mathcal{D}}_{m, \lambda} \frac{t^{m}}{m !}\right)\left(\sum_{k=0}^{\infty}\left(\sum_{l=0}^{k}(x)_{l, \lambda} S_{1, \lambda}(k, l)\right) \frac{t^{k}}{k !}\right) \\
& =\sum_{n=0}^{\infty}\left(\sum_{k=0}^{n} \sum_{l=0}^{k}\left(\begin{array}{l}
n \\
k
\end{array}\right)(x)_{l, \lambda} S_{1, \lambda}(k, l) \widetilde{\mathcal{D}}_{n-k, \lambda}\right) \frac{t^{n}}{n !} .
\end{aligned}
$$

By comparing the coefficients of the same powers of $t^{n}$ of (19) and (20), we arrive at the desired result.

Theorem 2.3 Let $n \in \mathbb{N}_{0}$. Then

$$
\beta_{n, \lambda}(x)=\sum_{m=0}^{n} \widetilde{\mathcal{D}}_{m, \lambda}(x) S_{2, \lambda}(n, m)
$$

Proof By changing $t$ to $e_{\lambda}(t)-1$ in (16) we see that

$$
\begin{aligned}
\sum_{m=0}^{\infty} \widetilde{\mathcal{D}}_{m, \lambda}(x) \frac{1}{m !}\left(e_{\lambda}(t)-1\right)^{m} & =\frac{t}{(1+\lambda t)^{\frac{1}{\lambda}}-1}(1+\lambda t)^{\frac{x}{\lambda}} \\
& =\sum_{n=0}^{\infty} \beta_{n, \lambda}(x) \frac{t^{n}}{n !} .
\end{aligned}
$$

In addition to expression (21), we have

$$
\begin{aligned}
\sum_{m=0}^{\infty} \widetilde{\mathcal{D}}_{m, \lambda}(x) \frac{1}{m !}\left(e_{\lambda}(t)-1\right)^{m} & =\sum_{m=0}^{\infty} \widetilde{\mathcal{D}}_{m, \lambda}(x) \sum_{m=n}^{\infty} S_{2, \lambda}(n, m) \frac{t^{n}}{n !} \\
& =\sum_{n=0}^{\infty}\left(\sum_{m=0}^{n} \widetilde{\mathcal{D}}_{m, \lambda}(x) S_{2, \lambda}(n, m)\right) \frac{t^{n}}{n !}
\end{aligned}
$$

Equating (21) and (22) proves the theorem. 
Theorem 2.4 The new type degenerate Daehee numbers of the second kind are computed by the recurrence relation

$$
\widetilde{\mathcal{D}}_{n, \lambda}(1)-\widetilde{\mathcal{D}}_{n, \lambda}= \begin{cases}0 & \text { if } n=0 \\ \lambda^{n-1}(1)_{n, 1 / \lambda} & \text { if } n \geq 1 .\end{cases}
$$

Proof We first consider

$$
\begin{aligned}
\sum_{n=1}^{\infty} \lambda^{n-1}(1)_{n, 1 / \lambda} \frac{t^{n}}{n !} & =\log _{\lambda}(1+t) \\
& \left.=\left(\left(1+\lambda \log _{\lambda}(1+t)\right)^{\frac{1}{\lambda}}-1\right)\right) \sum_{n=0}^{\infty} \widetilde{\mathcal{D}}_{n, \lambda} \frac{t^{n}}{n !} \\
& =\frac{\log _{\lambda}(1+t)}{\left(1+\lambda \log _{\lambda}(1+t)\right)^{\frac{1}{\lambda}}-1}\left(1+\lambda \log _{\lambda}(1+t)\right)^{\frac{1}{\lambda}}-\sum_{n=0}^{\infty} \widetilde{\mathcal{D}}_{n, \lambda} \frac{t^{n}}{n !} \\
& =\sum_{n=0}^{\infty}\left(\widetilde{\mathcal{D}}_{n, \lambda}(1)-\widetilde{\mathcal{D}}_{n, \lambda}\right) \frac{t^{n}}{n !} .
\end{aligned}
$$

By comparing the coefficients at $\frac{t^{n}}{n !}$ we complete the proof.

We now state the distribution formula.

Theorem 2.5 For $d \in \mathbb{N}$ and $n \geq 0$, we have

$$
\widetilde{\mathcal{D}}_{n, \lambda}(x)=\sum_{m=0}^{n} d^{m-1} S_{1, \lambda}(n, m) \sum_{a=0}^{d-1} \beta_{m, \frac{\lambda}{d}}\left(\frac{a+x}{d}\right) .
$$

Proof Using (16), we have

$$
\begin{aligned}
\sum_{n=0}^{\infty} \widetilde{\mathcal{D}}_{n, \lambda}(x) \frac{t^{n}}{n !} & =\frac{\log _{\lambda}(1+t)}{\left(1+\lambda \log _{\lambda}(1+t)\right)^{\frac{1}{\lambda}}-1}\left(1+\lambda \log _{\lambda}(1+t)\right)^{\frac{x}{\lambda}} \\
& =\frac{\log _{\lambda}(1+t)}{\left(1+\lambda \log _{\lambda}(1+t)\right)^{\frac{d}{\lambda}}-1} \sum_{a=0}^{d-1}\left(1+\lambda \log _{\lambda}(1+t)\right)^{\frac{a+x}{\lambda}} \\
& =\frac{1}{d}\left(\frac{d \log _{\lambda}(1+t)}{\left(1+\frac{\lambda}{d}\left(d \log _{\lambda}(1+t)\right)\right)^{\frac{d}{\lambda}}-1}\right) \sum_{a=0}^{d-1}\left(1+\frac{\lambda}{d}\left(d \log _{\lambda}(1+t)\right) \frac{\left(\frac{a+x}{d}\right)}{d}\right. \\
& =\frac{1}{d} \sum_{a=0}^{d-1} \sum_{m=0}^{\infty} \beta_{m, \frac{\lambda}{d}}\left(\frac{a+x}{d}\right) \frac{1}{m !}\left(d \log _{\lambda}(1+t)\right)^{m} \\
& =\frac{1}{d} \sum_{a=0}^{d-1} \sum_{m=0}^{\infty} \beta_{m, \frac{\lambda}{d}}\left(\frac{a+x}{d}\right) \sum_{n=m}^{\infty} d^{m} S_{1, \lambda}(n, m) \frac{t^{n}}{n !} \\
& =\sum_{n=0}^{\infty}\left(\sum_{m=0}^{n} \sum_{a=0}^{d-1} d^{m-1} \beta_{m, \frac{\lambda}{d}}\left(\frac{a+x}{d}\right) S_{1, \lambda}(n, m)\right) \frac{t^{n}}{n !}
\end{aligned}
$$

where $d \in \mathbb{N}$ and $n \geq 0$. By (25) and (26) we arrive at the desired result. 
Theorem 2.6 For $n \in \mathbb{N}, m \geq 0$, we have

$$
\frac{1}{m+1}\left(\widetilde{\mathcal{D}}_{m+1, \lambda}(n)-\widetilde{\mathcal{D}}_{m+1, \lambda}\right)=\sum_{j=0}^{m} \sum_{k=0}^{j} \sum_{l=0}^{n-1}\left(\begin{array}{c}
m \\
j
\end{array}\right)(l)_{k, \lambda} S_{1, \lambda}(j, k) D_{m-j, \lambda}
$$

Proof We first consider the following expression:

$$
\begin{aligned}
\log _{\lambda}(1+t) \sum_{l=0}^{n-1}\left(\left(1+\lambda \log _{\lambda}(1+t)\right)^{\frac{1}{\lambda}}\right)^{l} \\
=\frac{\log _{\lambda}(1+t)}{\left(1+\lambda \log _{\lambda}(1+t)\right)^{\frac{1}{\lambda}}-1}\left(1+\lambda \log _{\lambda}(1+t)\right)^{\frac{n}{\lambda}}-\frac{\log _{\lambda}(1+t)}{\left(1+\lambda \log _{\lambda}(1+t)\right)^{\frac{1}{\lambda}}-1} \\
=\sum_{m=0}^{\infty}\left(\widetilde{\mathcal{D}}_{m, \lambda}(n)-\widetilde{\mathcal{D}}_{m, \lambda}\right) \frac{t^{n}}{n !} \\
=t \sum_{m=0}^{\infty}\left(\frac{\widetilde{\mathcal{D}}_{m+1, \lambda}(n)-\widetilde{\mathcal{D}}_{m+1, \lambda}}{m+1}\right) \frac{t^{m}}{m !} .
\end{aligned}
$$

Now we proceed (27) with different perspective as follows:

$$
\begin{aligned}
& =t\left(\frac{\log _{\lambda}(1+t)}{t}\right) \sum_{l=0}^{n-1}\left(1+\lambda \log _{\lambda}(1+t)\right)^{\frac{l}{\lambda}} \\
& =t\left(\sum_{p=0}^{\infty} d_{p, \lambda} \frac{t^{p}}{p !}\right)\left(\sum_{j=0}^{\infty}\left(\sum_{k=0}^{j} \sum_{l=0}^{n-1}(l)_{k, \lambda} S_{1, \lambda}(j, k)\right) \frac{t^{j}}{j !}\right) \\
& =t \sum_{m=0}^{\infty}\left(\sum_{j=0}^{m} \sum_{k=0}^{j} \sum_{l=0}^{n-1}\left(\begin{array}{c}
m \\
j
\end{array}\right)(l)_{k, \lambda} S_{1, \lambda}(j, k) d_{m-j, \lambda}\right) \frac{t^{m}}{m !} .
\end{aligned}
$$

By comparing the coefficients at $\frac{t^{n}}{n !}$ of (27) and (29) we complete the proof.

We now consider a new type of higher-order degenerate Daehee polynomials of the second kind.

Definition 2.2 Let $r$ be a positive integer. New type higher-order degenerate Daehee polynomials of the second kind are defined by the following generating function:

$$
\sum_{n=0}^{\infty} \widetilde{\mathcal{D}}_{n}^{(r)}(x) \frac{t^{n}}{n !}=\left(\frac{\log _{\lambda}(1+t)}{\left(1+\lambda \log _{\lambda}(1+t)\right)^{\frac{1}{\lambda}}-1}\right)^{r}\left(1+\lambda \log _{\lambda}(1+t)\right)^{\frac{x}{\lambda}} .
$$

When $x=0, D_{n, \lambda}^{(r)}=: D_{n, \lambda}^{(r)}(0)$ are called new type higher-order degenerate Daehee polynomials of the second kind.

It is worth noting that

$$
\lim _{\lambda \rightarrow 0} \widetilde{\mathcal{D}}_{n, \lambda}^{(r)}(x)=D_{n}^{(r)}(x)
$$

representing the Daehee polynomials of higher order. 
Theorem 2.7 Let $n$ be a nonnegative integer. Then we have the following summation formula:

$$
\widetilde{\mathcal{D}}_{n, \lambda}^{(r)}(x)=\sum_{m=0}^{n} \beta_{m, \lambda}^{(r)}(x) S_{1, \lambda}(n, m) .
$$

Proof Using (30), we have

$$
\begin{aligned}
\sum_{n=0}^{\infty} \widetilde{\mathcal{D}}_{n, \lambda}^{(r)}(x) \frac{t^{n}}{n !} & =\left(\frac{\log _{\lambda}(1+t)}{\left(1+\lambda \log _{\lambda}(1+t)\right)^{\frac{1}{\lambda}}-1}\right)^{r}\left(1+\lambda \log _{\lambda}(1+t)\right)^{\frac{x}{\lambda}} \\
& =\sum_{m=0}^{\infty} \beta_{m, \lambda}^{(r)}(x) \frac{1}{m !}\left(\log _{\lambda}(1+t)\right)^{m} \\
& =\sum_{m=0}^{\infty} \beta_{m, \lambda}^{(r)}(x) \sum_{n=m}^{\infty} S_{1, \lambda}(n, m) \frac{t^{n}}{n !} \\
& =\sum_{n=0}^{\infty}\left(\sum_{m=0}^{n} \beta_{m, \lambda}^{(r)} S_{1, \lambda}(n, m)\right) \frac{t^{n}}{n !},
\end{aligned}
$$

where we have used the following series manipulation:

$$
\sum_{m=0}^{\infty} a_{m} \sum_{n=m}^{\infty} b_{n}=\sum_{n=0}^{\infty} \sum_{m=0}^{n} a_{m} b_{n} .
$$

Since (32) $=(33)$, we arrive at the desired result.

Theorem 2.8 Let $n$ be a natural number. Then the inversion formula of (31) is given by the following relation:

$$
\beta_{m, \lambda}^{(r)}(x)=\sum_{m=0}^{n} \widetilde{\mathcal{D}}_{m, \lambda}^{(r)} S_{2, \lambda}(n, m) .
$$

Proof By replacing $t$ by $e_{\lambda}(t)-1$ in (30) we get

$$
\begin{aligned}
\sum_{m=0}^{\infty} \widetilde{\mathcal{D}}_{m, \lambda}^{(r)}(x) \frac{1}{m !}\left(e_{\lambda}(t)-1\right)^{m} & =\left(\frac{t}{(1+\lambda t)^{\frac{1}{\lambda}}-1}\right)^{r}(1+\lambda t)^{\frac{x}{\lambda}} \\
& =\sum_{n=0}^{\infty} \beta_{n, \lambda}^{(r)}(x) \frac{t^{n}}{n !}
\end{aligned}
$$

On the other hand, we see that

$$
\begin{aligned}
\sum_{m=0}^{\infty} \widetilde{\mathcal{D}}_{m, \lambda}^{(r)}(x) \frac{1}{m !}\left(e_{\lambda}(t)-1\right)^{m} & =\sum_{m=0}^{\infty} \widetilde{\mathcal{D}}_{m, \lambda}^{(r)}(x) \sum_{n=m}^{\infty} S_{2, \lambda}(n, m) \frac{t^{n}}{n !} \\
& =\sum_{n=0}^{\infty}\left(\sum_{m=0}^{n} \widetilde{\mathcal{D}}_{m, \lambda}^{(r)}(x) S_{2, \lambda}(n, m)\right) \frac{t^{n}}{n !}
\end{aligned}
$$

Matching (34) and (35) proves the theorem. 
Theorem 2.9 Let the variables $r$ and $k$ be natural numbers with $r>k$. Then we have the following summation formula:

$$
\widetilde{\mathcal{D}}_{n, \lambda}^{(r)}(x)=\sum_{l=0}^{n}\left(\begin{array}{l}
n \\
l
\end{array}\right) \widetilde{\mathcal{D}}_{l, \lambda}^{(r-k)} \widetilde{\mathcal{D}}_{n-l, \lambda}^{(k)}(x) \quad(n \geq 0) .
$$

Proof Since

$$
\sum_{n=0}^{\infty} \widetilde{\mathcal{D}}_{n, \lambda}^{(r)}(x) \frac{t^{n}}{n !}=\left(\frac{\log _{\lambda}(1+t)}{\left(1+\lambda \log _{\lambda}(1+t)\right)^{\frac{1}{\lambda}}-1}\right)^{r}\left(1+\lambda \log _{\lambda}(1+t)\right)^{\frac{x}{\lambda}}
$$

we have

$$
\begin{aligned}
& =\left(\frac{\log _{\lambda}(1+t)}{\left(1+\lambda \log _{\lambda}(1+t)\right)^{\frac{1}{\lambda}}-1}\right)^{r-k}\left(\frac{\log _{\lambda}(1+t)}{\left(1+\lambda \log _{\lambda}(1+t)\right)^{\frac{1}{\lambda}}-1}\right)^{k}\left(1+\lambda \log _{\lambda}(1+t)\right)^{\frac{x}{\lambda}} \\
& =\left(\sum_{l=0}^{\infty} \widetilde{\mathcal{D}}_{l, \lambda}^{(r-k)} \frac{t^{l}}{l !}\right)\left(\sum_{m=0}^{\infty} \widetilde{\mathcal{D}}_{m, \lambda}^{(k)}(x) \frac{t^{m}}{m !}\right) \\
& =\sum_{n=0}^{\infty}\left(\sum_{l=0}^{n}\left(\begin{array}{c}
n \\
l
\end{array}\right) \widetilde{\mathcal{D}}_{l, \lambda}^{(r-k)} \widetilde{\mathcal{D}}_{n-l, \lambda}^{(k)}(x)\right) \frac{t^{n}}{n !} .
\end{aligned}
$$

Equating (36) and (37) proves the theorem.

It is well known from [17] that

$$
\left(\frac{t}{\log _{\lambda}(1+t)}\right)^{k}(1+t)^{x-1}=\sum_{n=0}^{\infty} B_{n, \lambda}^{(n-k+1)}(x) \frac{t^{n}}{n !} \quad(k \in \mathbb{Z})
$$

where $B_{n, \lambda}^{(\alpha)}(x)$ are called $\lambda$-analogue higher-order Bernoulli polynomials, which are given by the generating function

$$
\left(\frac{t}{\lambda e^{t}-1}\right)^{\alpha} e^{x t}=\sum_{n=0}^{\infty} B_{n, \lambda}^{(\alpha)}(x) \frac{t^{n}}{n !}
$$

Theorem 2.10 Let $n$ be a natural number. Then we have the following explicit summation formula:

$$
\widetilde{\mathcal{D}}_{n}^{(r)}(x)=\sum_{m=0}^{n} B_{m, \lambda}^{(m-k+1)}\left(\frac{x}{\lambda}+1\right) S_{1, \lambda}(n, m) .
$$

Proof Changing the parameter $t$ to $\log _{\lambda}(1+t)$ and $x$ to $\frac{x}{\lambda}+1$ in (38) gives

$$
\begin{aligned}
& \sum_{m=0}^{\infty} B_{m, \lambda}^{(m-k+1)}\left(\frac{x}{\lambda}+1\right) \frac{\left(\log _{\lambda}(1+t)\right)^{m}}{m !} \\
& \quad=\left(\frac{\log _{\lambda}(1+t)}{\left(1+\lambda \log _{\lambda}(1+t)\right)^{\frac{1}{\lambda}}-1}\right)^{r}\left(1+\lambda \log _{\lambda}(1+t)\right)^{\frac{x}{\lambda}}
\end{aligned}
$$




$$
=\sum_{n=0}^{\infty} \widetilde{\mathcal{D}}_{n}^{(r)}(x) \frac{t^{n}}{n !}
$$

By (39) we have

$$
\begin{aligned}
\sum_{m=0}^{\infty} B_{m, \lambda}^{(m-k+1)}\left(\frac{x}{\lambda}+1\right) \frac{\left(\log _{\lambda}(1+t)\right)^{m}}{m !} & =\sum_{m=0}^{\infty} B_{m, \lambda}^{(m-k+1)}\left(\frac{x}{\lambda}+1\right) \sum_{n=m}^{\infty} S_{1, \lambda}(n, m) \frac{t^{n}}{n !} \\
& =\sum_{n=0}^{\infty}\left(\sum_{m=0}^{n} B_{m, \lambda}^{(m-k+1)}\left(\frac{x}{\lambda}+1\right) S_{1, \lambda}(n, m)\right) \frac{t^{n}}{n !}
\end{aligned}
$$

Matching the coefficients at $\frac{t^{n}}{n !}$ in Eqs. (40) and (41), we obtain

$$
\widetilde{\mathcal{D}}_{n}^{(r)}(x)=\sum_{m=0}^{n} B_{m, \lambda}^{(m-k+1)}\left(\frac{x}{\lambda}+1\right) S_{1, \lambda}(n, m) .
$$

This completes the proof of the theorem.

In the case where $x=0$ in Theorem 2.10, we have

$$
\widetilde{\mathcal{D}}_{n}^{(r)}=\sum_{m=0}^{n} B_{m, \lambda}^{(m-k+1)}(1) S_{1, \lambda}(n, m)
$$

Theorem 2.11 Let $n$ be a natural number. We have the following summation formula:

$$
\widetilde{\mathcal{D}}_{n, \lambda}^{(r)}(x)=\sum_{k=0}^{n} \sum_{m=0}^{k}\left(\begin{array}{l}
n \\
k
\end{array}\right)(x)_{m, \lambda} S_{1, \lambda}(k, m) \widetilde{\mathcal{D}}_{n-k, \lambda}^{(r)} .
$$

Proof Using (30), we have

$$
\begin{aligned}
\sum_{n=0}^{\infty} \widetilde{\mathcal{D}}_{n, \lambda}^{(r)}(x) \frac{t^{n}}{n !} & =\left(\frac{\log _{\lambda}(1+t)}{\left(1+\lambda \log _{\lambda}(1+t)\right)^{\frac{1}{\lambda}}-1}\right)^{r}\left(1+\lambda \log _{\lambda}(1+t)\right)^{\frac{x}{\lambda}} \\
& =\left(\sum_{l=0}^{\infty} \widetilde{\mathcal{D}}_{l, \lambda}^{(r)} \frac{t^{l}}{l !}\right)\left(\sum_{k=0}^{\infty}\left(\sum_{m=0}^{k}(x)_{m, \lambda} S_{1, \lambda}(k, m)\right) \frac{t^{k}}{k !}\right) \\
& =\sum_{n=0}^{\infty}\left(\sum_{k=0}^{n} \sum_{m=0}^{k}\left(\begin{array}{l}
n \\
k
\end{array}\right)(x)_{m, \lambda} S_{1, \lambda}(k, m) \widetilde{\mathcal{D}}_{n-k, \lambda}^{(r)}\right) \frac{t^{n}}{n !} .
\end{aligned}
$$

This completes the proof of the theorem.

Theorem 2.12 Let $n$ be a natural number. Then the following addition property for $D_{n, \lambda}^{(r)}(x)$ holds:

$$
\widetilde{\mathcal{D}}_{n, \lambda}^{(r)}(x+y)=\sum_{k=0}^{n} \sum_{m=0}^{k}\left(\begin{array}{l}
n \\
k
\end{array}\right) \widetilde{\mathcal{D}}_{n-k, \lambda}^{(r)}(x) S_{1, \lambda}(k, m)(y)_{m, \lambda} .
$$


Proof Observe that

$$
\begin{aligned}
\sum_{n=0}^{\infty} \widetilde{\mathcal{D}}_{n, \lambda}^{(r)}(x+y) \frac{t^{n}}{n !} & =\left(\frac{\log _{\lambda}(1+t)}{\left(1+\lambda \log _{\lambda}(1+t)\right)^{\frac{1}{\lambda}}-1}\right)^{r}\left(1+\lambda \log _{\lambda}(1+t)\right)^{\frac{x+y}{\lambda}} \\
& =\left(\sum_{l=0}^{\infty} \widetilde{\mathcal{D}}_{l, \lambda}^{(r)}(x) \frac{t^{l}}{l !}\right)\left(\sum_{k=0}^{\infty}\left(\sum_{m=0}^{k}(y)_{m, \lambda} S_{1, \lambda}(k, m)\right) \frac{t^{k}}{k !}\right) \\
& =\sum_{n=0}^{\infty}\left(\sum_{k=0}^{n} \sum_{m=0}^{k}\left(\begin{array}{l}
n \\
k
\end{array}\right) \widetilde{\mathcal{D}}_{n-k, \lambda}^{(r)}(x) S_{1, \lambda}(k, m)(y)_{m, \lambda}\right) \frac{t^{n}}{n !}
\end{aligned}
$$

By equating (42) and (43) we complete the proof of the theorem.

Theorem 2.13 Let $n$ be a natural number. Then we have following formula:

$$
\widetilde{\mathcal{D}}_{n, \lambda}^{(-r)}=\sum_{k=0}^{n} \sum_{l=0}^{k} \frac{\left(\begin{array}{c}
n \\
k
\end{array}\right)}{\left(\begin{array}{c}
(+r \\
k
\end{array}\right)} S_{2, \lambda}(l+r, r) S_{1, \lambda}(k+r, l+r) B_{n-k, \lambda}^{(n-k-r+1)}(1) .
$$

Proof Using (38), we have

$$
\begin{aligned}
\sum_{n=0}^{\infty} \widetilde{\mathcal{D}}_{n, \lambda}^{(-r)} \frac{t^{n}}{n !} & =\left(\frac{\left(1+\lambda \log _{\lambda}(1+t)\right)^{\frac{1}{\lambda}}-1}{\log _{\lambda}(1+t)}\right)^{r} \\
& =\left(\frac{t}{\log _{\lambda}(1+t)}\right)^{r} \frac{r !}{t^{r}} \frac{1}{r !}\left(\left(1+\lambda \log _{\lambda}(1+t)\right)^{\frac{1}{\lambda}}-1\right)^{r} \\
& =\left(\sum_{m=0}^{\infty} B_{m, \lambda}^{(m-r+1)}(1) \frac{t^{m}}{m !}\right) \frac{r !}{t^{r}}\left(\sum_{l=r}^{\infty} S_{2, \lambda}(l, r) \frac{1}{l !}\left(\log _{\lambda}(1+t)\right)^{l}\right) \\
& =\left(\sum_{m=0}^{\infty} B_{m, \lambda}^{(m-r+1)}(1) \frac{t^{m}}{m !}\right) \frac{r !}{t^{r}}\left(\sum_{l=0}^{\infty} S_{2, \lambda}(l+r, r) \frac{1}{(l+r) !}\left(\log _{\lambda}(1+t)\right)^{l+r}\right) \\
& =\left(\sum_{m=0}^{\infty} B_{m, \lambda}^{(m-r+1)}(1) \frac{t^{m}}{m !}\right) \frac{r !}{t^{r}}\left(\sum_{k=r}^{\infty}\left(\sum_{l=0}^{k-r} S_{2, \lambda}(l+r, r) S_{1, \lambda}(k, l+r)\right) \frac{t^{k}}{k !}\right) \\
& =\left(\sum_{m=0}^{\infty} B_{m, \lambda}^{(m-r+1)}(1) \frac{t^{m}}{m !}\right)\left(\sum_{k=0}^{\infty}\left(\sum_{l=0}^{k} \frac{S_{2, \lambda}(l+r, r) S_{1, \lambda}(k+r, l+r)}{\left(\begin{array}{c}
k+r \\
k
\end{array}\right)}\right) \frac{t^{k}}{k !}\right) \\
& =\sum_{n=0}^{\infty}\left\{\sum_{k=0}^{n} \sum_{l=0}^{k} \frac{\left(\begin{array}{c}
n \\
k
\end{array}\right)}{\left(\begin{array}{c}
(+r \\
k
\end{array}\right)} S_{2, \lambda}(l+r, r) S_{1, \lambda}(k+r, l+r) B_{n-k, \lambda}^{(n-k-r+1)}(1)\right\} \frac{t^{n}}{n !} .
\end{aligned}
$$

This completes the proof of the theorem.

\section{Conclusions}

The idea of degenerate traces back to Carlitz [1]. The aim of degenerate version is to introduce new special functions, polynomials, and numbers. This is one of ways of introducing new type special functions, polynomials, and numbers. Another way of introducing new special functions, polynomials, and numbers is studying $q$-analogs of special polynomials. As has been seen in the references, Kim and his research team $([2-17,19-21])$ have 
been intensively studying degenerate versions of new special functions, polynomials, and numbers. In this paper, motivated by the works of Kim and his research team, we have studied new type degenerate versions of Daehee numbers and polynomials of the second kind. In this paper, we have derived their explicit, closed, and summation formulae using their generating functions, series manipulation, and analytical means. It seems that these types of polynomials will be continued to be studied due to their interesting reflections in the fields of mathematics, statistics, and sciences.

\section{Acknowledgements}

We would like to thank the reviewers and Associate Editor Prof. Dr. Taekyun Kim for valuable suggestions and comments, which have substantially improved the presentation of the paper.

\section{Funding}

The authors extend their appreciation to the Deanship of Scientific Research at Majmaah University for funding this work under project number (RGP-2019-25).

\section{Availability of data and materials}

Not applicable.

\section{Competing interests}

The authors declare that they have no competing interests.

\section{Authors' contributions}

All authors contributed equally to this papers. All authors read and approved the final manuscript.

\section{Author details}

'Department of Information System, College of Computer and Information Sciences, Majmaah University, Majmaah 11952, Saudi Arabia. ${ }^{2}$ Department of Mathematics and Natural Sciences, Prince Mohammad Bin Fahd University, P.O. Box: 1664, Al Khobar 31952, Saudi Arabia. ${ }^{3}$ Department of Economics, Faculty of Economics, Administrative and Social Sciences, Hasan Kalyoncu University, TR-27410 Gaziantep, Turkey. ${ }^{4}$ College of Engineering, Majmaah University, Majmaah 11952, Saudi Arabia.

\section{Publisher's Note}

Springer Nature remains neutral with regard to jurisdictional claims in published maps and institutional affiliations.

Received: 27 May 2020 Accepted: 12 August 2020 Published online: 18 August 2020

\section{References}

1. Carlitz, L.: Degenerate Stirling, Bernoulli and Eulerian numbers. Util. Math. 15, 51-88 (1979)

2. Dolgy, D.V., Jang, G.-W., Kim, D.S., Kim, T.: Explicit expressions for Catalan-Daehee numbers. Proc. Jangjeon Math. Soc. 20(1), 1-9 (2017)

3. Jang, L.-C., Kim, W., Kwon, V., Kim, T.: On degenerate Daehee polynomials and numbers of the third kind. J. Comput. Appl. Math. 364, 112343 (2020)

4. Khan, W.A., Nisar, K.S., Duran, U., Acikgoz, M., Araci, S.: Multifarious implicit summation formulae of Hermite-based poly-Daehee polynomials. Appl. Math. Inf. Sci. 12(2), 305-310 (2018)

5. Kim, B.M., Yun, S.J., Park, J.-W.: On a degenerate $\lambda$-q-Daehee polynomials. J. Nonlinear Sci. Appl. 9(6), 4607-4616 (2016)

6. Kim, D.S., Kim, T.: Some $p$-adic integrals on $\mathbb{Z}_{p}$ associated with trigonometric functions. Russ. J. Math. Phys. 25(3), 300-308 (2018)

7. Kim, D.S., Kim, T.: A note on polyexponential and unipoly functions. Russ. J. Math. Phys. 26(1), 40-49 (2019)

8. Kim, D.S., Kim, T.: A note on a new type of degenerate Bernoulli numbers. Russ. J. Math. Phys. 27(2), 227-235 (2020)

9. Kim, D.S., Kim, T., Kwon, H.I., Jang, G.-W.: Degenerate Daehee polynomials of the second kind. Proc. Jangjeon Math. Soc. 21(1), 83-97 (2018)

10. Kim, T., Jang, L.-C., Kim, D.S., Kim, H.Y.: Some identities on Type 2 degenerate Bernoulli polynomials of the second kind. Symmetry 12(4), $510(2020)$

11. Kim, T., Kim, D.S.: Degenerate Laplace transform and degenerate gamma function. Russ. J. Math. Phys. 24(2), 241-248 (2017)

12. Kim, T., Kim, D.S.: Identities involving degenerate Euler numbers and polynomials arising from non-linear differential equations. J. Nonlinear Sci. Appl. 9, 2086-2098 (2016)

13. Kim, T., Kim, D.S.: A note on type 2 Changhee and Daehee polynomials. Rev. R. Acad. Cienc. Exactas Fís. Nat., Ser. A Mat. 113(3), 2783-2791 (2019)

14. Kim, T., Kim, D.S.: Degenerate polyexponential functions and degenerate Bell polynomials. J. Math. Anal. Appl. 487(2), $124017(2020)$

15. Kim, T., Kim, D.S.: Degenerate binomial coefficients and degenerate hypergeometric functions. Adv. Differ. Equ. 2020, Article ID 115 (2020)

16. Kim, T., Kim, D.S.: A note on central Bell numbers and polynomials. Russ. J. Math. Phys. 27(1), 76-81 (2020) 
17. Kim, T., Kim, D.S., Kim, H.-Y., Kwon, J.: A new type degenerate Daehee numbers and polynomials (2020) 19 Apr 2020. arXiv:2004.08743v1 [math.NT]

18. Kim, T., Kim, D.S., Kim, H.Y., Jang, L.-C.: Degenerate poly-Bernoulli numbers and polynomials. Informatica 31(3), 2-8 (2020)

19. Kim, T., Kim, D.S., Kim, H.Y., Kwon, J.: Degenerate Stirling polynomials of the second kind and some applications. Symmetry 11(8), Article ID 1046 (2019)

20. Kim, T., Kim, D.S., Kim, H.Y., Kwon, J.: Some results on degenerate Daehee and Bernoulli numbers and polynomials. Adv. Differ. Equ. 2020, 311 (2020)

21. Kwon, J., Kim, T., Kim, D.S., Kim, H.Y.: Some identities for degenerate complete and incomplete $r$-Bell polynomials. J. Inequal. Appl. 2020, Article ID 23 (2020)

22. Kwon, J., Kim, W.J., Rim, S.-H.: On the some identities of the type 2 Daehee and Changhee polynomials arising from p-adic integrals on $\mathbb{Z}_{p}$. Proc. Jangjeon Math. Soc. 22(3), 487-497 (2019)

23. Lee, J.G., Kwon, J., Jang, G.-W., Jang, L.-C.: Some identities of $\lambda$-Daehee polynomials. J. Nonlinear Sci. Appl. 10(8), 4137-4142 (2017)

24. Roman, S.: The Umbral Calculus. Pure and Applied Mathematics, vol. 111. Academic Press, New York (1984)

25. Simsek, Y.: Identities on the Changhee numbers and Apostol-type Daehee polynomials. Adv. Stud. Contemp. Math. (Kyungshang) 27(2), 199-212 (2017)

Submit your manuscript to a SpringerOpen ${ }^{\circ}$ journal and benefit from:

- Convenient online submission

- Rigorous peer review

- Open access: articles freely available online

- High visibility within the field

- Retaining the copyright to your article

Submit your next manuscript at $\boldsymbol{\triangleright}$ springeropen.com 